cise in outline. The bulk of the soft tissue consisted of oral granular cells, more resembling tubercle than anything else, except that the outline was too sharp. There mere, besides free granular matter, large glomeruli or " inHammation corpuscles", and free oil. The same description applies to the microscopy of the creamy juice obtained from the mass.

While, then, wo without any hesitation would insist upon the propriety of classing the growth among the malignants, we may advert to this instance as a further proof that we cannot by the microscope alone establish any definition which may comprise all cancerous growths. Like so many other iustances of disease, it is the complex of symptoms, and not the individual feature, by which our diagnosis must be determined.

The cortex of the left kidney was found to be entirely destroyed; but here the deposit presented quite the characteristics of cancer: and this is the more curious, as the kidney was manifestly secondarily affected.

The affection of the heart, curious as it was in a diagnostic point of view, was equally so in regard to its pathology ; for, in addition to the molecular changes in the myolemma, there was a deposit in the muscular tissue, which, under the circumstances, could scarcely be regarded in any light but as analogous to the morbid growth found in other parts, but varying in form and size with the organ.

\section{CASE OF ABDOMINAL ANEURISM, WITH OBSERVATIONS UPON ITS DIFFERENTIAL DIAGNOSIS.}

By C. M. Dirrant, MI.D., Physician to the East Suffolk and Ipswich Iospital.

Or the history of the present case, I know but little. He was a fine made man, of between forty and fifty years of age, and a serjeant-major in the militia. It appears that he had suffered from pain in his back for about thirteen months; and the regiment having been ordered about this time to wear the shell jacket instead of the coat, he, perhaps naturally, although of course erroneously, ascribed his pain, and subsequent illness, in part, at least, to this cause. From that period he had, I believe, suffered more or less pain, although it was not sufficient to prevent him fulfilling the harassing and fatiguing duties attendant upon an encampment.

The pain, both in the back and right hypochondrium, extending down to the groin and thigh, became latterly most agonising; and nothing short of almost constant narcotism, from morphia, tended to afford the slightest mitigation. I am not aware when the pulsation in the abdomen was first discovered, but as an interesting feature in connexion with the autopsy, during the last week of his life, all pulsation had nearly ceased, and he sank gradually, but the pain, at times excruciating, continued to the end.

Autopsy. The body was emaciated, and on opening the abdomen, a large mass could be felt, extending from a little below the diaphragm, downwards, into the right iliac region.

On closer examination, an aneurismal sac, the size of a large orange, and filled with firm fibrin, was found to spring from the aorta, about two inches below the diaphragm. This sac had evidently gradually given way, as a still larger tumour, containing semi-organised coagulum, was found within the folds of the mesentery, which indeed had formed its envelope, and which, on tracing it upwards, was seen to proceed from the original aneurismal sac. On examining the vertebral canal, the bodies of two of the dorsal were found to be almost entirely removed by absorption. The cessation of pulsation in the tumour, which had previously been strong, may be readily explained by the rupture and gradual escape of the liquid portion of the contents of the primary sac, which had become nearly filled by fibrinous deposit. The connexion of the eccondary effusion within the mesentery with the original aneurismal pouch was very distinctly demonstrable.

REXARKS. Ventral aneurism is characterised by the existence of a pulsating tumour, of variable extent, in the course of the abdominal aorta. To the hand, the sensation afforded is that of a fixed, rounded, more or less compressible mass, giving a full, swelling, expansive, as well as heaving pulsation, and sometimes accompanied by a purring tremor. The chief impulse is generally to the left of the spine, but not always, as the sac occasionally enlarges only to the right side, where alone the impulse is there felt. The force of the shock received by the hand is often quite out of proportion to the size of the aneurismal sac, while, in other instances, we may have a large sac, unaccompanied by either impulse or murmur. The size of the tumour may, in general, be defined by careful percussion; but this is not always practicable, as the limit is sometimes obscured by flatulent distension of the intestines.

On applying the stethoscope we may hear an abrupt, short, moderately loud, and deeply seated systolic bellows murmur, less harsh and grating than in thoracic ancurism, and which is sometimes more marked in the loft lumbar region than in front. The murmur may be dull and muffled, requiring the pressure of the stethoscope to elicit its distinct character. It is iimited transversely to the seat of the disease, and propagated more extensively below than above that spot, and where sometimes it is alone audible. Dr. Walshe mentions, and which I have once had an opportunity of verifying, that, in some instances, systolic murmur is audible in the reclining, when inaudible in the erect posture; and, again, as already stated, that, in other cases, no murmur whaterer can be detceted in any posture. Lastl 5 , the aneurismal sound is inaudible orer the heart.

The reneral symptoms of ventral aortic aneurism, when taken per se, are extremely vague and perplexing.

The vessel, in its passage through the abdomen, being so closely embraced by filaments from the solar plexus, and other nerves, the neuralgic sufferings are often very intense. The seat of the pain, often intermittent in character, is, of course, influenced by the locality of the sac; it is especially severe in the loins, lancinating upwards and in front, to the hypochoudriac and iliac regions, and below, to the testicles and lower extremities, which may become contracted and convulsed. The pain often assumes the character of colic, and the poor patient describes his sufferings in the most graphic terms. The pulse is not often materially affected, and the appetite often continues moderately good. Prcssure on the intestines may interfere with the functions of these viscera; and if the sac be large and seated high up in the abdomen, it may, by impcding phrenic action, render the respiration accelerated, and give rise to severe pulmonary distress.

Such are the leading features, physical and general, which, more or less, obtain in aneurism of the abdominal aorta, and we may now briefly consider those other affections by which this is occasionally simulated, and which become both important and interesting in reference to its differential diagnosis.

Ventral aneurism is sometimes closely resembled by simple aortic pulsation, arising from functional disturbance. This latter, occurring in dyspeptic and anæmic subjects, will be recognised by its concomitant nerrous or anæmic symptoms. In aneurism, the pain is sometimes truly agonising; in simple aortic pulsation, this symptom is absent. The functional disorder will be further diagnosed by its aggravation on slight exciting causes, and by its want of permanency. Again, the sound and impulse is short, abrupt, and jerking, in contradistinction to the slow, heaving, expansive pulsation of aneurism, nor is the impulse so decidedly confined to a particular portion of the artery.

Inorganic pulsation may be attended with a systolic sound; but this, as a rule, is inaudible in the back. It is not unfrequently accompanied by a coexisting murmur in the neck.

Lastly, the sex of the patient will assist the diagnosis; 
for while abdominal aneurism in the fomale is of very rare cocurrence, inorganic aortic pulsation is very common. Preal accumulation and impaction has been mistaken for aneurism of the abdominal aorta. The oval shape of the tumour, with its doughy, inelastic feel, and the result of free purgation, will, in general, suffice to clear up the diagnosis.

Fnlarged glands, or organic disease of the abdominal viscera, either lying over, or pressing laterally upon the artery, will sometimes closely simulate aneurism. The history of the cases, and especially the existence of malignant disease in other parts of the body will aid our opinion; while in pulsation from superimposed tumours, the murmur may often be interrupted by change of position, or by applying the stethoscope laterally, und displacing the tumour from off the artery. The obscurity of symptoms and pain accompanying lumbar and psoas abscess may resemble the phenomena of aneurisin; but the swelling, elongating downwards, without sound or impulse, and the want of the globular tumour of the enlarged artery, will, in general, distinguish these affections.

Tumours of the kidney may, by extending irregularly inwards, and receiving the shock of the artery, sometimes resemble an aneurismal swelling; but the condition of the urine, and previous history of the case, will sufficiently assist the diagnosis. It should be borne in mind, however, that an aneurism pressing upon the emulgent vein may render the urine albuminous. In all cases in which the symptoms are obscure-and in very many they will inevitably prove at first to be so-it will be advisable to examine the patient both in the erect, horizontal, and lateral postures, and, in some instances, it may be prudent to watch the case for a time before risking an opinion.

\section{CASE OF ARM AND SHOULDER PRESENTATION IN WHICH EVISCERATION WAS PERFORMED.}

By Robert Manners Mann, Esq., Manchester.

As cases requiring an operation of this description are of rare occurrence, $I$ think the publication of the following in our JourNAI may prove interesting, as well as instructive, to some of our readers.

Case. On Thursday evening, April 3rd, I received an urgent message to attend Mrs. L., aged 22 years, who was said to be in premature labour of her first child. On arriving at her residence, I found her suffering from tolerably strong and expulsive uterine pains. An examination per vaginam informed me that a considerable portion of the bag of membranes was protruded through the os uteri into the ragina, and all but without the os externum. - Neither during a pain nor in the intcrvals between, could I detect any presentation; but I discovered high up ths os uteri, and the not as yet obliterated cervix, shewing me that she was between her sixth and seventh month. The os was perhaps so far dilated as to be capable of receiving two or three fingers.

Strong expulsive efforts continuing, the membranes soon gave way; and I now expected some portion of the foetus to follow shortly. In this, however, I was disappointed; for, although there was an immediate and considerable fow of liquor amnii and much dribbling afterwards, I could only just by pressing my finger firmly upwards perceive that there was a limb of the fotus, but whether an arm or leg, could not make out to my satisfaction. The pains now ceased entirely ; and after waiting some time, I advised my patient to get up, and think no more about it, until the action of the uterus should again be excited. I left strict orders to be sent for on the return of pains, as I have often known delivery take place very suddenly in such premature cases, even in primipara. No message, howerer, came for me till Saturday morning, April 5th, at eight o'clock.

I now found her having both strong and frequent pains; and on examining, discovered the right hand, arm, and shoulder, presenting, together with \& very dry, unjielding, and undilatable state of the soft parts. The pains continued to increase in violence; and as the state of parts would not as yet justify me in attempting to turn, I resigned myself patiently to the hope, that as the foetus was premature, and consequently softer and more yielding, that nature's efforts would suffice, or that the possible but unusual circumstance of spontaneous evolution might occur. After waiting a few hours, however, the shoulder only got lower, and the chest more and more firmly wedged in the pelvic carity; but in the mcan time the os uteri was becoming more dilated, and I therefore decided upon making an attempt to turn. Using lard freely, I then in the usual way, but with much difficulty, succeeded in passing $\mathbf{m y}$ left hand into the ragina, and afterwards, by long continued pressure through a rigid and strongly resisting os uteri, managed to insinuate it into the uterus. Owing, however, to the two circumstances of the liquor amnii having been so long evacuated, and the vigorous muscular action going on cramping my hand, it was with considerable difficulty that I at length reached what proved afterwards to be the right foot. I could not by any efforts I dare use bring this down more than just barely to shew itself through the labia majora. A slip noose was then made in some tape, and passed over the foot and secured round the ankle, but still all traction failed to make any change, and the arm kept slipping down as fast as I returned it. My patient had suffered very severely, and was getting exhausted, so that after maturc consideration, I decided that evisceration of the fotus would not only afford her speedy relief, but would be the only means left for her delivery. My perforating instruments were soon brought, and I got an assistant to hold the arm and employ traction by it, for me to steady the chest; after which, I was enabled easily to evacuate the contents of the thorax and alsdomen, and directly afterwards hooked my finger in the pelvis, brought down the legs, and without much more trouble the head followed.

There was no doubt whatever in my mind that the fœtus had been dead for a considerable period previous to the operation. The pelvis is diminutive. My patient is now (April 16th) sitting up, and progressing in every way extremely well.

\section{A CASE OF STRANGULATED FEMORAL HERNIA, WITH PROTRUSION OF THE INTESTINE ON THE FIFTH DAY : RECOVERY.}

By T. M. WARD, Esq., Exmouth.

CAsE. Mrs. E. H., aged 66 years, a spare but tolerably healthy woman, has fallen under my care several times during the last two years, with strangulated femoral hernia. I have always had considerable difficulty in returning the bowel. The tumour was large and hard, and the symptoms very urgent. In spite of my repeated ro monstrances, she neglected to wear a truss regularly; and in this state, with a severe cough, she walked on the 10th of February to Lympstone, two miles from home. While there, the hernia descended, and becamc strangulated. My friend Mr. Price, of that place, was called in, and after a long and patient trial of the taxis, failed in reducing it. He then gave her an injection with a flexible tube, and a pill with three grains of opium, repeating a grain dose every hour. When under the influence of the opiate, he tried the taxis upon her again, unsuccessfully.

On the morning of the 1Jth, she was brought home, and placed under my care, when I repeated the attempt, with a like result. Mr. Price then coming to my aid, we made her inhale chloroform, and then we both made a final but fruitless effort to return the hernia.

Having explained to her the necessity of an immediate operation, to which she at once consented, I procured the kind aid of my friend Mr. Eustace (a retired surgeon), an : at 3 P.x. proceeded to operate. Mr. Price kept her all ine time well under the influence of chloroform, and also afforaed 